\title{
Review: relying on published data alone overestimates efficacy and safety of SSRIs in children
}

Whittington CJ, Kendall T, Fonagy $P$, et al. Selective serotonin reuptake inhibitors in childhood depression: systematic review of published versus unpublished data. Lancet 2004;363:1341-5. Does the risk-benefit profile of selective serotonin reuptake inhibitors (SSRIs) in the treatment of depression in children
change when unpublished data is added to the meta-analysis?

\section{METHODS}

$\square$

Design: Systematic review with meta-analysis

Data sources: EMBASE, MEDLINE, PsycINFO, CINAHL, and the

Cochrane Library searched from inception to April 2003, plus

hand searches of key journals, previous systematic reviews and meta-analyses, and contact with experts. Unpublished data extracted from a Committee on Safety of Medicines report on the use of SSRIs in children with depression.

11

Study selection and analysis: Inclusion criteria: English language RCTs published in peer reviewed journals, or reviewed in the Committee on Safety of Medicines report comparing SSRI with placebo for depression in young people (aged 5-18 years). The quality of each RCT was assessed. Trials not clearly described as randomised were excluded. Tests for heterogeneity were carried out. Risk-benefit profiles were calculated for each SSRI based on relative and absolute statistics.

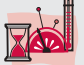

Outcomes: Remission (Children's Depression Rating Scalerevised (CDRS-R) score $<29$; Hamilton Depression Rating Scale $<8)$; response to treatment ( $50 \%$ reduction in CDRS-R score); changes in mean depression level (CDRS-R); serious adverse events; suicidal behaviour and attempts; number of participants discontinuing treatment

\section{MAIN RESULTS}

See http://www.ebmentalhealth.com/supplemental for table for RR of SSRIs versus placebo.

Fluoxetine: two published RCTs $(\mathrm{n}=315)$ plus unpublished data $(\mathrm{n}=458)$ met inclusion criteria. Fluoxetine significantly increased remission and improved treatment response compared with placebo. Fewer children reported serious adverse effects (SAEs) with fluoxetine compared with placebo. Data on suicidal behaviour were not reported in published trials; unpublished data showed no increased risk with fluoxetine.

Paroxetine: one published RCT $(\mathrm{n}=180)$ plus two unpublished trials $(n=478)$ met inclusion criteria. Paroxetine increased remission compared with placebo but there were no significant differences in response or reduction in depressive symptoms between groups. Paroxetine increased SAEs compared with placebo. In the unpublished trials, paroxetine did not significantly improve outcomes compared with placebo. Combining data revealed no significant differences between groups, except for SAEs, which were significantly more likely with paroxetine.

Sertraline: two published RCTs $(\mathrm{n}=376)$ plus unpublished data from these trials met inclusion criteria. Sertraline improved response compared with placebo but there were no significant differences in reduction in depressive symptoms between groups.

For correspondence: Craig Whittington, Centre for Outcomes Research and Effectiveness, Subdepartment of Clinical Health Psychology, University College London, Torrington Place, London, UK; c.whittington@ucl.ac.uk Sources of funding: NICE (National Institute for Clinical Excellence).
Citalopram: Only data from two unpublished trials $(\mathrm{n}=422)$ met inclusion criteria. Citalopram did not significantly improve depressive symptoms compared with placebo. Citalopram was associated with a small increase in SAEs compared with placebo.

Venlafaxine: one RCT $(\mathrm{n}=40)$ plus two unpublished trials $(\mathrm{n}=334)$ met inclusion criteria. Published data indicated that venlafaxine did not significantly improve depressive symptoms; no SAEs were reported. With unpublished data, venlafaxine was associated with an increased risk of suicide related behaviour and an increased risk of discontinuing treatment due to adverse effects.

\section{CONCLUSIONS}

Fluoxetine is likely to be beneficial for depression in young people, and has a favourable risk-benefit profile compared with other SSRIs. Citalopram and venlafaxine are unlikely to be beneficial. For paroxetine, sertraline, and venlafaxine, published data alone indicate that benefits may outweigh the risks; however, combining published and unpublished data suggests that the risks outweigh the benefits. Non-publication of trials and data can lead to false assumptions regarding efficacy of treatments.

\section{Commentory}

7 he article raises significant issues about how the efficacy and risks of treatment are evaluated. Many meta-analytic reviews only include studies that have been published in peer reviewed journals. This approach uses the peer review process as a quality control measure. However, the Whittington et al study shows the limitation in this process because the bias of most journals is to publish mostly studies with positive findings. In addition, in a competitive environment, pharmaceutical companies are also more reluctant to share negative findings. The analysis that Whittington and his colleagues provide demonstrates how including negative but not published studies can significant change the analysis of the safety and efficacy of several serotonin reuptake inhibitors (SSRI) in treating children with depressive disorders.

The inclusion of unpublished studies poses challenges. The first challenge is gaining access to the studies. As the authors suggest, a process including both cooperation and regulation would be helpful to provide greater access to all pharmaceutical studies for medications and new devices that have been licensed for clinical use. However it would also be helpful to have access to governmental and foundation funded studies that are not published.

The second challenge is obtaining sufficient information to evaluate the quality of the research. Journals are not uniform about the information included in their articles. It is sometimes difficult for individuals attempting to review existing studies to have sufficient access to the information. It would be helpful for reports to include uniform information about the studies so that it is possible for reviewers to evaluate the quality of the research.

The current review shows a lack of sufficient efficacy and safety with regards to the use of SSRI in children and adolescents with the exception of fluoxetine. However, it is important to note that the findings are not definitive and more rigorous study may result in a revision of the current findings.

Mark L Wolraich, MD

CMRI/Shaun Walters Professor of Pediatrics, OU Child Study Center, Oklahoma City, OK, USA 